Article

\title{
Tunable Mid-Infrared Graphene Plasmonic Cross-Shaped Resonator for Demultiplexing Application
}

\author{
Somayyeh Asgari * ${ }^{-10}$ and Tapio Fabritius \\ Optoelectronics and Measurement Techniques Research Unit, Faculty of Information Technology and Electrical \\ Engineering, University of Oulu, 90570 Oulu, Finland.; tapio.fabritius@oulu.fi \\ * Correspondence: somayyeh.asgari@oulu.fi
}

Received: 30 December 2019; Accepted: 6 February 2020; Published: 10 February 2020

check for updates

\begin{abstract}
In this study, a tunable graphene plasmonic filter and a two-channel demultiplexer are proposed, simulated, and analyzed in the mid-infrared (MIR) region. We discuss the optical transmission spectra of the proposed cross-shaped resonator and the two-channel demultiplexer. The transmission spectra of the proposed MIR resonator are tunable by change of its dimensional parameters and the Fermi energy of the graphene. Our proposed structures have a single mode in the wavelength range of 5-12 $\mu \mathrm{m}$. The minimum full width at half maximum (FWHM) and the maximum transmission ratio of the proposed resonator respectively reached $220 \mathrm{~nm}$ and $55 \%$. Simulations are performed by use of three-dimensional finite-difference time-domain (3D-FDTD) method. Coupled mode theory (CMT) is used to investigate the structure theoretically. The numerical and the theoretical results are in good agreement. The performance of the proposed two-channel demultiplexer is investigated based on its crosstalk. The minimum value of crosstalk reaches $-48.30 \mathrm{~dB}$. Our proposed structures are capable of providing sub-wavelength confinement of light waves, useful in applications in MIR region.
\end{abstract}

Keywords: graphene; plasmonics; Fermi energy; resonator; demultiplexer

\section{Introduction}

Surface plasmon polaritons (SPPs) are evanescent electromagnetic surface waves, shorter in wavelength than the incident light wave, which travel along the metal-dielectric. The terms of 'surface plasmon' and 'polariton' are respectively involved charge motion in highly conductive matter, and electromagnetic waves in the dielectric or vacuum. The materials which are frequently used in plasmonic structures are typically metals such as silver and gold, which are exploited in various applications such as filters [1-3], demultiplexers [4-6], sensors [7-9], and switches [10-12].

The parameters of the dielectric constants of metals are challengeable to be adjusted and controlled in different wavelengths [13-15]. In comparison, graphene is a two-dimensional (2D) sheet which dielectric constant can be modulated dynamically by alternation of its applied bias voltage enabling unique features to be utilized in mid-infrared (MIR) region [16-20]. In practice, graphene has strong confinement of lightwaves, long propagation length, and low loss making it superior to metals [21-23]. Because of the significant advantages of graphene, many graphene based structures-such as filters [24,25], demultiplexers [26,27], modulators [28,29], couplers [30,31], absorbers [32,33], and solar cells [34]-are designed and analyzed. Fabrication of graphene-based devices in MIR region was recently reported in $[35,36]$. The graphene/SiO2 MIR resonators are compatible with CMOS filters and resonators $[37,38]$ making them attractive.

Previously published works $[4,5,24,26,39-48]$ have reported transmission ratios up to $99 \%$ and FWHM values between 2-650 $\mathrm{nm}$. In the design of demultiplexers, it is important to minimize 
the cross talk, thus the aim of this study was to find a design for demultiplexer which presents a better cross talk comparing to $[4,5,26,39,44-46]$. Moreover, a demultiplexer design should be conducted so that it maintains a desired single mode resonance in a wider wavelength range than previously reported structures $[4,5,24,26,39,40,42-48]$.

Plasmonic metal-based cross-shaped resonator and its demultiplexing application are investigated previously in [4]. The proposed cross-shaped resonator in [4] is single mode in the near-infrared wavelength range of $400 \mathrm{~nm}$ to $2 \mu \mathrm{m}$ while the proposed single mode resonator is designed to work at the wider MIR wavelength range. In addition, the transmission spectra of our structures aimed to be tunable since the Fermi energy of graphene can be changed without needing to re-fabricate the structures. Moreover, the cross talk of the graphene-type demultiplexer is improved compared to the metal-based one. To the best of our knowledge, tunable graphene cross-shaped resonator side-coupled to another resonator with the function of wavelength selecting and demultiplexing has not been studied yet.

In this manuscript, a new kind of plasmonic two-channel demultiplexer based on input/output graphene waveguides and cross-shaped resonator is proposed for (MIR) region. The resonator is investigated by coupled mode theory (CMT) and three-dimensional finite-difference time-domain (3D-FDTD) methods. By changing Fermi level of graphene, the length of the resonator, and the width of the resonator, the resonance wavelengths can be tuned. First, we study the transmission of the cross-shaped resonator. Then, two resonators are designed and integrated into one structure to achieve two-channel demultiplexer structure. The advantages of our designed structures are their nanometer dimensions which would be used in future MIR devices and photonic integrated circuits. The proposed concept provides an interesting and useful route for implementing photonic structures for MIR region.

\section{Resonator and Analysis}

Two-dimensional top and side views of the proposed graphene plasmonic MIR resonator are shown in Figure 1a,b. The structure is composed of a cross-shaped resonator embedded between the horizontal input and vertical output graphene waveguides. The whole structure is placed on $\mathrm{SiO}_{2} / \mathrm{Si}$. $\mathrm{SiO}_{2}$ is used as a buffering layer and it has significant influence on the transmission spectrum of the proposed MIR resonator. Previous studies have reported that the thickness of $\mathrm{SiO}_{2}$ cannot be lower than $50 \mathrm{~nm}$ due to the invariant transmission caused by the fabrication errors and faults $[49,50]$. Two Au electrodes are used for applying the bias voltage to graphene in the proposed concept.

The materials selection and the device design are made so that they are reasonable from the fabrication point of view. The $\mathrm{Au}$ film and $\mathrm{SiO}_{2} / \mathrm{Si}$ spacer can be deposited by using the conventional electron beam evaporation technique [51]. Then, the monolayer graphene can be coated on the top of the $\mathrm{SiO}_{2}$ spacer after a chemical vapor deposition (CVD). Finally, the cross-shaped graphene MIR resonator with its input and output waveguides can be fabricated by using electron beam lithography and oxygen plasma etching [17].

The incident electromagnetic lightwave is launched to the resonator from its input port in MIR region and SPPs are excited along the input graphene waveguide. Two monitors are used at $P_{\text {in }}$ and $P_{\text {out }}$ to monitor the input and output powers. The input and output monitors are respectively 2D $X$-normal and 2D Y-normal. The transmission is calculated by $T=\frac{P_{\text {out }}}{P_{\text {in }}}$.

Only the incident wavelength, which satisfies the resonance condition of the cross-shaped resonator, can be coupled effectively to the resonator and pass through to the output port while the others are suppressed. Thus, the band pass filtering effect is obtained in MIR region. Structural and material parameters of the proposed graphene plasmonic resonator are given in Table 1. Numerical simulations are performed by use of three-dimensional finite-difference time-domain (3D-FDTD) method in Lumerical FDTD Software package under appropriate boundary conditions of perfectly matched layers (PML). The assumed mesh sizes of the proposed resonator in three different directions 
of $x, y$, and $z$ are given in Table 2. The optical conductivity of graphene $\sigma_{G}$, is expressed by the Kubo formula [22]

$$
\sigma_{G}=\frac{2 i e^{2} k_{B} T}{\pi \hbar^{2}\left(\omega+i \tau^{-1}\right)} \ln \left[2 \cosh \left(\frac{E_{f}}{2 k_{B} T}\right)\right]+\frac{i e^{2}}{4 \pi \hbar} \ln \left[\frac{2 E_{f}-\left(\omega+i \tau^{-1}\right) \hbar}{2 E_{f}+\left(\omega+i \tau^{-1}\right) \hbar}\right]
$$

where $e, k_{B}, T, \hbar, \omega, E_{f}$, and $\tau$ are respectively the electron charge, Boltzmann constant, temperature, reduced Planck constant, angular frequency, and Fermi energy level, and carrier relaxation time. $\tau$ can be calculated by $\tau=\frac{\mu E_{f}}{e v_{f}^{2}}$, where $\mu$ is the carrier mobility. The constant $v_{f}=10^{6} \mathrm{~m} / \mathrm{s}$ is the Fermi velocity of graphene.

As depicted in Figure 1b, graphene should be biased by external gate voltage. $E_{f}$ is related to the bias voltage $V_{\text {bias }}$ as [52]

$$
E_{f}=\sqrt{\frac{\pi \varepsilon_{0} \varepsilon_{r S i O 2} V_{b i a s}}{e t_{S i O 2}}}
$$

where $\varepsilon_{0}$ is the permittivity of air with the value of $1 . \varepsilon_{r S i O 2}$ and $t_{\mathrm{SiO} 2}$ are respectively the relative permittivity and the thickness of the buffer layer. Moreover, the permittivity of graphene can be determined by

$$
\varepsilon_{G}=1+\frac{i \sigma_{G}}{\varepsilon_{0} \omega t}
$$

where $t$ is the thickness of graphene and is assumed to be $1 \mathrm{~nm}$.

In the air-graphene-air system, the dispersion relation of SPPs travelled on graphene can be represented by [53]

$$
\beta_{S P P}=k_{0} \sqrt{1-\left(\frac{2}{\eta_{0} \sigma_{G}}\right)^{2}}
$$

where $\beta_{S P P}, k_{0}$, and $\eta_{0}$ are respectively the propagation constant of SPPs, the wave vector of incident light, and the impedance of air.

The transfer function $t f$, of the proposed graphene plasmonic resonator structure by use of coupled mode theory (CMT) method is [54]

$$
t f=\frac{\frac{1}{Q_{w}}}{j 2 \delta+\frac{1}{Q_{i}}+\frac{1}{Q_{w}}}
$$

where $Q_{w}$ and $Q_{i}$ are the quality factors of the resonator which are respectively describing the waveguide coupling loss and the intrinsic loss. $\delta$ is used for normalization of the frequency $\omega$ and is defined as $\delta=\frac{\omega-\omega_{0}}{\omega_{0}}$. Transmission $T$ is obtained by $T=|t f|^{2}$ [54].

Transmission spectrum of the MIR graphene plasmonic resonator of Figure 1 obtained by numerical FDTD and theoretical CMT methods are given in Figure 2a. For the transmission spectrum which is obtained by CMT method, $Q_{w}$ and $Q_{i}$ are respectively considered as 40 and 100. $|E|$ Field profiles of the resonator for the resonance wavelength of $\lambda=7.5 \mu \mathrm{m}$ and the non-resonance wavelength of $\lambda=6.5 \mu \mathrm{m}$ are respectively given in Figure $2 \mathrm{~b}$,c. The SPPs are excited by use of a dipole point source with electric polarization which is considered $2 \mathrm{~nm}$ above the horizontal input graphene [55]. In the resonance wavelength, the incident lightwave couples effectively to the resonator and then, it decouples to the output graphene waveguide. In the non-resonance wavelength, the incident lightwave cannot couple to the resonator and it is blocked.

The real part of the $\beta_{S P P}$ is related to the $\sigma_{G}$ (Equation (4)) and $\sigma_{G}$ is related to the $\mathrm{E}_{\mathrm{f}}$ (Equation (1)). As the $\mathrm{E}_{\mathrm{f}}$ increases, the real part of the $\beta_{S P P}$ decreases (depicted in Figure $2 \mathrm{~d}$ ). The wavelength of the transmission peak shown in Figure 2a should thus exhibit a blue shift when the Fermi energy increases. That is how the transmission spectrum can be tuned by the change of the Fermi energy of graphene. 


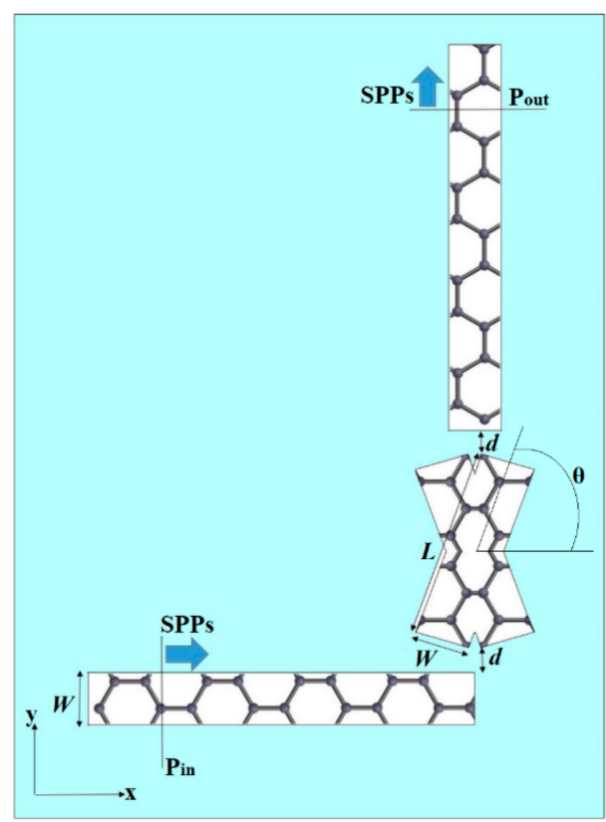

(a)

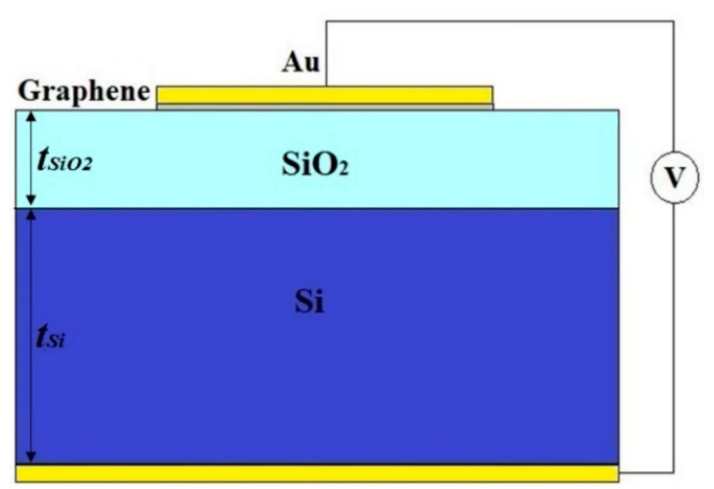

(b)

Figure 1. Two-dimensional (2D) (a) top and (b) side views of the MIR cross-shaped graphene plasmonic resonator. Two input/output graphene waveguides are coupled by a graphene cross-shaped resonator.

Table 1. Structural and material parameters of the proposed graphene plasmonic resonator of Figure 1.

\begin{tabular}{ccc}
\hline Parameter & Label & Value \\
\hline Resonator length & $L$ & $90 \mathrm{~nm}$ \\
\hline Resonator width & $W$ & $30 \mathrm{~nm}$ \\
\hline Angle between the ribbon and the horizontal line in the cross-shaped resonator & $\theta$ & $40^{\circ}$ \\
\hline Distance between the input/output waveguide and the resonator & $d$ & $10 \mathrm{~nm}$ \\
\hline Graphene Fermi energy & $E f$ & $0.8 \mathrm{eV}$ \\
\hline Thickness of SiO2 & $t S i O 2$ & $50 \mathrm{~nm}$ \\
\hline Thickness of Si & $t S i$ & $500 \mathrm{~nm}$ \\
\hline Refractive index of SiO2 & $n S i O 2$ & $\sqrt{2.1}$ \\
\hline Refractive index of Si & $n S i$ & $\sqrt{11.08}$ \\
\hline
\end{tabular}




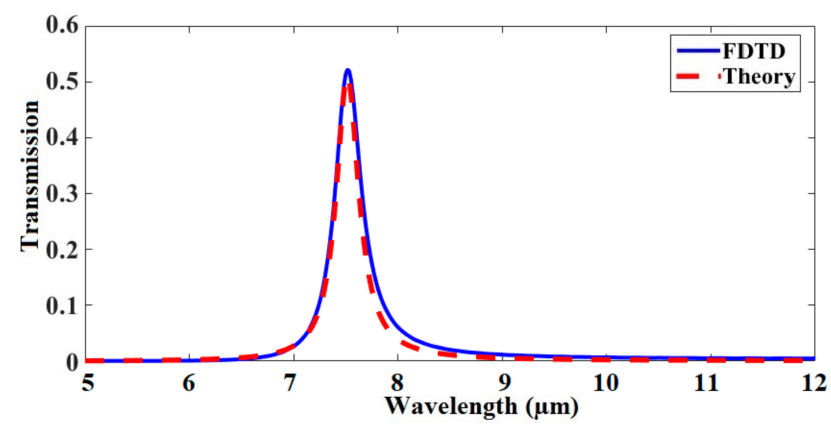

(a)

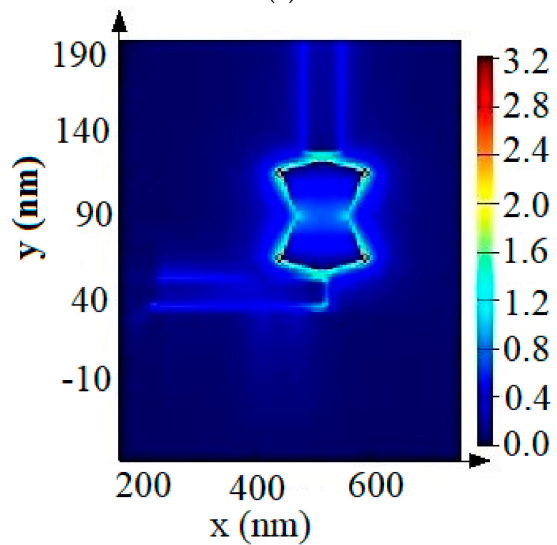

(b)

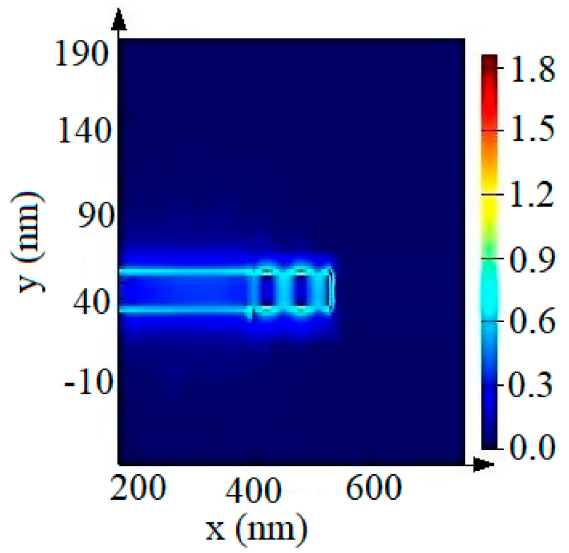

(c)

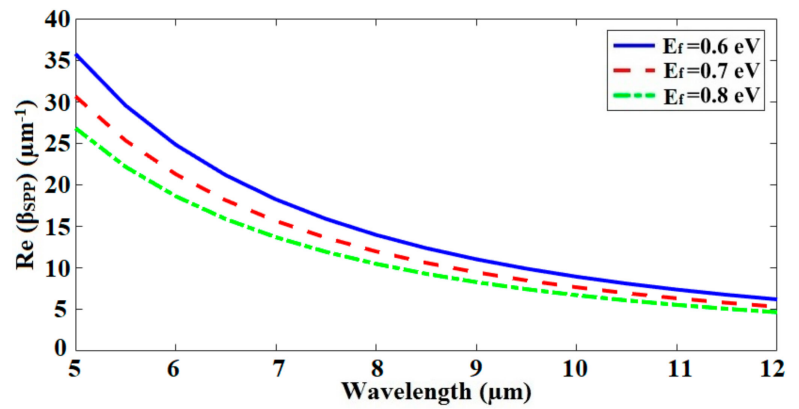

(d)

Figure 2. (a) Transmission spectrum of the resonator structure of Figure 1 obtained by numerical FDTD and theoretical CMT methods. $|E|$ Field profiles of the resonator structure of Figure 1 for $(\mathbf{b})$ the resonance wavelength of $\lambda=7.5 \mu \mathrm{m}$, (c) the non-resonance wavelength of $\lambda=6.5 \mu \mathrm{m}$, and (d) the dependence of the real part of the $\beta_{S P P}$ (Equation (4)) of the graphene layer for different Fermi energies of graphene. 
Table 2. Assumed mesh sizes of the proposed resonator structure of Figure 1.

\begin{tabular}{cccc}
\hline Segment/Direction & $\mathbf{x}(\mathbf{n m})$ & $\mathbf{y}(\mathbf{n m})$ & $\mathbf{z}(\mathbf{n m})$ \\
\hline Input waveguide & 6 & 2 & 0.1 \\
\hline Resonator & 2 & 2 & 0.1 \\
\hline Output waveguide & 2 & 6 & 0.1 \\
\hline
\end{tabular}

The resonance condition of the proposed resonator of Figure 1 could be expressed by [56]

$$
2 \beta_{S P P} L+2 \delta=2 p \pi
$$

where $\beta_{S P P}=\frac{2 \pi n_{S P P}}{\lambda}, n_{S P P}$ and $\lambda$ are respectively the effective refractive index of SPPs and the resonance wavelength. The phase change at the ends of the ribbon is denoted by $\delta$ and $p$ which are integer numbers. While the incident wavelength is much larger than the ribbon length, we can assume $p=1$. Therefore, the resonance wavelength could be given by [56]

$$
\lambda=\frac{2 \pi n_{S P P} L}{\pi-\delta}
$$

Transmission spectra of the proposed graphene plasmonic resonator of Figure 1 for three different values of the resonator length $\mathrm{L}$, the Fermi energy $\mathrm{E}_{\mathrm{f}}$, the resonator width $\mathrm{W}$, and the angle between the ribbon and the horizontal line of the cross-shaped resonator $\theta$ are simulated and given in Figure $3 a-d$, respectively.

As depicted in Figure 3a, by increasing the resonator length, $\mathrm{L}$, the resonance wavelength increases which shows a red shift. By Equation (7), it is clear there is a direct relationship between the resonance wavelength and the resonator length. Therefore, by increasing of the resonator length, the resonance wavelength increases.

By increasing of $E_{f}$, as shown in Figure $3 b$, the resonance wavelength decreases causing a blue shift. It is because the real part of the $\beta_{S P P}$ (Equation (4)) decreases as the $E_{\mathrm{f}}$ increases (given in Figure $2 \mathrm{~d}$ ). At the same time, the losses of the SPP wave supported by the graphene layer decrease with the $E_{\mathrm{f}}$ increase. Therefore, the transmission of the transmission peak increases with the $\mathrm{E}_{\mathrm{f}}$ increase, also discussed in $[57,58]$.

Real part of $n_{S P P}$ depends on the width of the graphene ribbon $W$. For $W<<L$, by decreasing of $W$, the real part of $n_{S P P}$ increases. Therefore, the transmission spectrum of the resonator experiences a blue shift by change of $W$ when $W<<L$, also discussed in [56,59]. By increasing $W$, as seen in Figure 3(c), the resonance wavelength decreases which shows a blue shift.

As can be seen from Equation (7), when the rotation angle between the graphene ribbon and the horizontal line $\theta$ changes, the length of the resonator $L$ is constant, while the effective refractive index $n_{S P P}$ will change slightly due to the variation of the coupling strength affected by change of $\theta$. By increasing of $\theta$, the coupling strength increases. Therefore, the real part of $n_{S P P}$ increases which is also discussed in [60]. Therefore, by increasing of $\theta$, the resonance wavelength tends to exhibit a blue shift (shown in Figure 3d). 


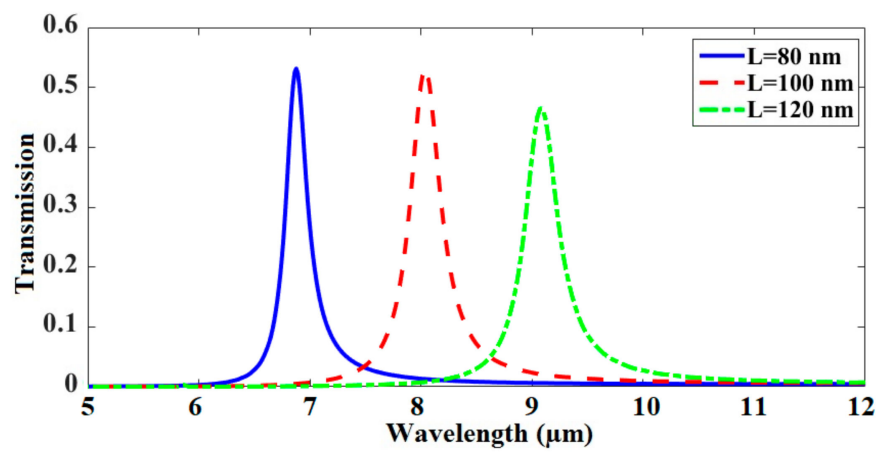

(a)

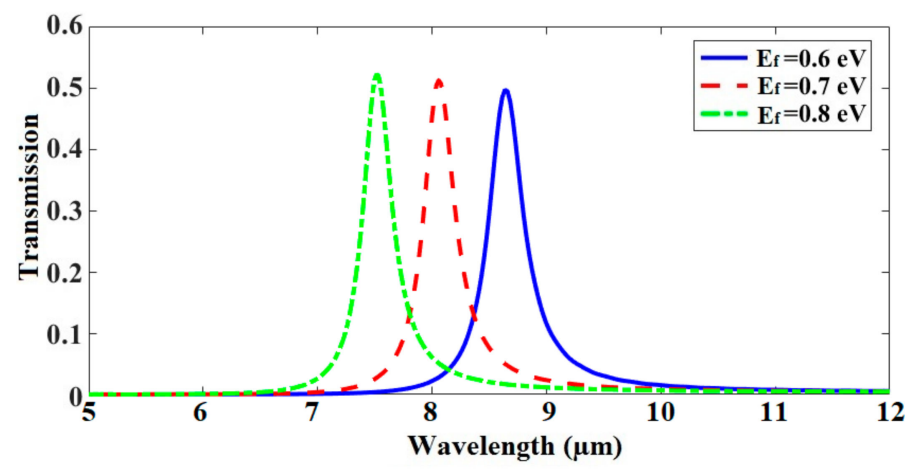

(b)

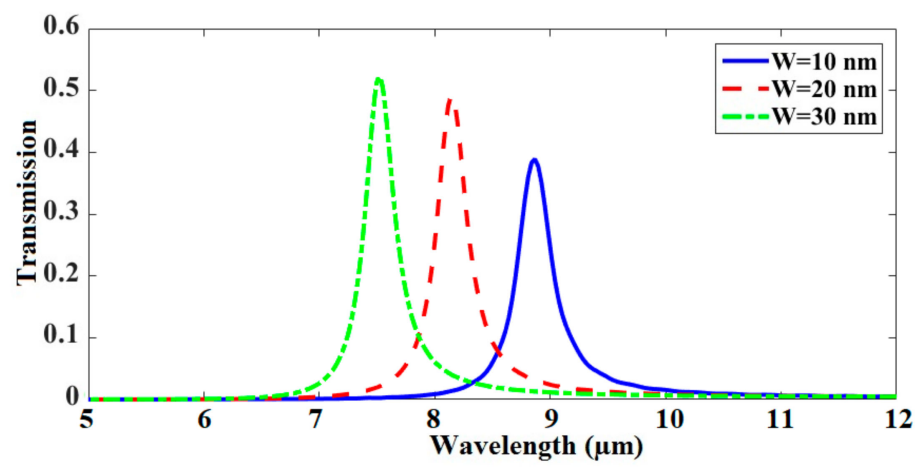

(c)

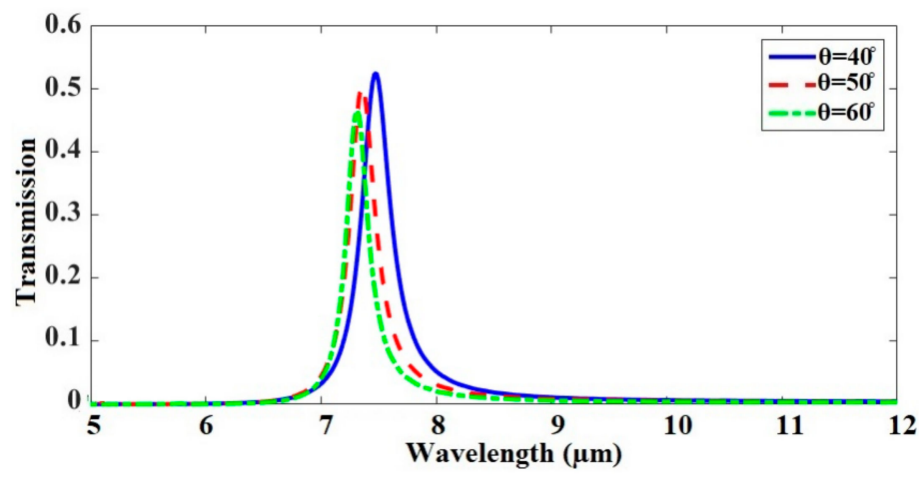

(d)

Figure 3. The influence of the alternation of (a) resonator length $L,(b)$ graphene Fermi energy $E_{f}$, (c) resonator width $\mathrm{W}$, and (d) the angle between the ribbon and the horizontal line of the resonator $\theta$, on the transmission spectrum of the proposed structure of Figure 1. 


\section{Two-Channel Demultiplexer and Analysis}

By modifying the cross-shaped resonator described in previous section, we propose and analyze a two-channel plasmonic demultiplexer in this section. The $2 \mathrm{D}$ view of two-channel plasmonic demultiplexer is depicted in Figure 4a. Transmission spectra of the two output channels of the demultiplexer are presented in Figure $4 \mathrm{~b}-\mathrm{e}$. The differences of the resonators of Figure $4 \mathrm{~b}-\mathrm{e}$ are respectively the lengths, Fermi energies, widths, and angles. Other parameters are the same as reported in Table 1. Electric field profiles of the proposed demultiplexer for the resonators with different lengths in the resonance wavelengths of $\lambda=6.8 \mu \mathrm{m}$ and $\lambda=8.05 \mu \mathrm{m}$ are respectively presented in Figure $4 \mathrm{f}, \mathrm{g}$.

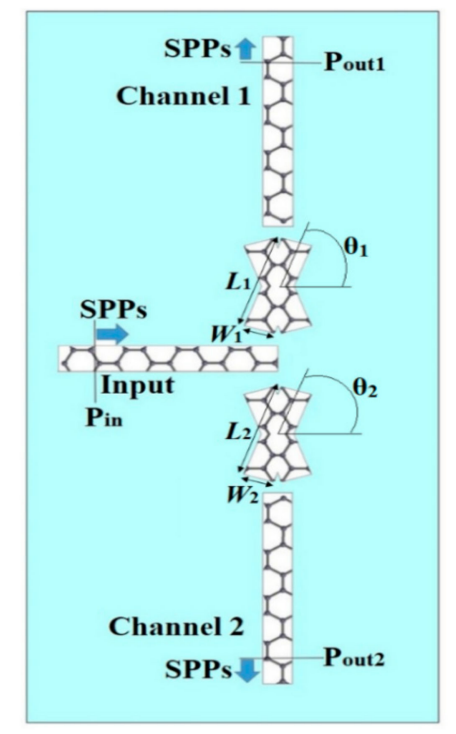

(a)

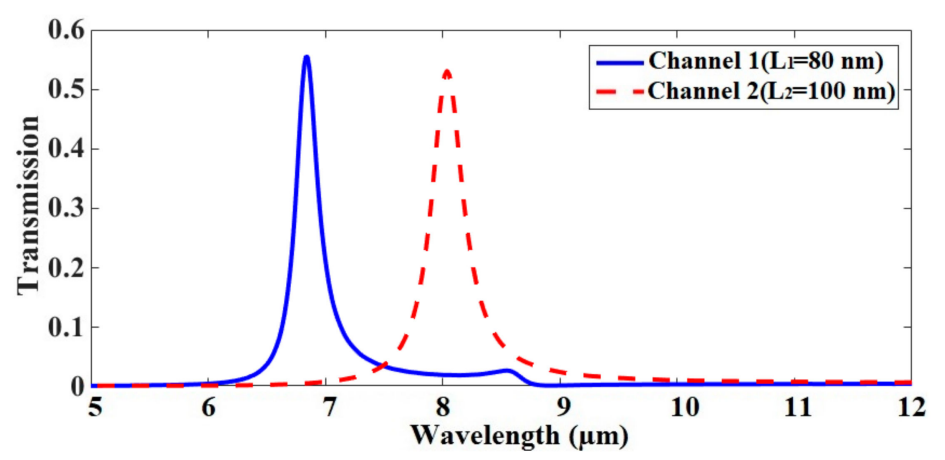

(b)

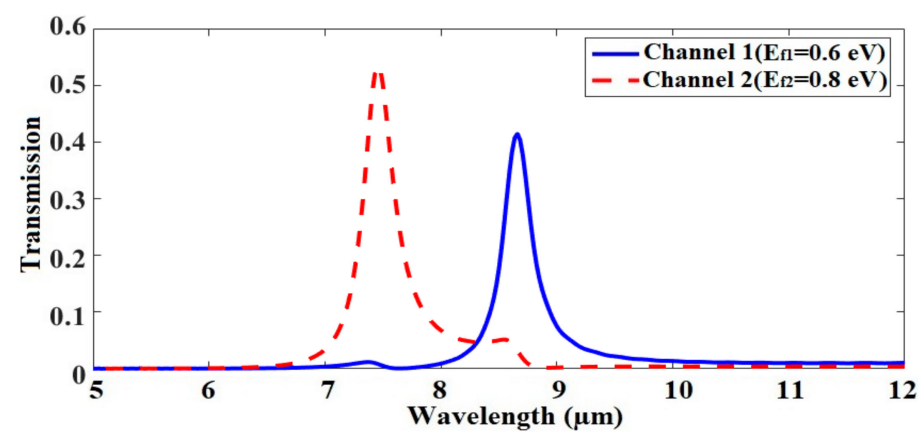

(c)

Figure 4. Cont . 


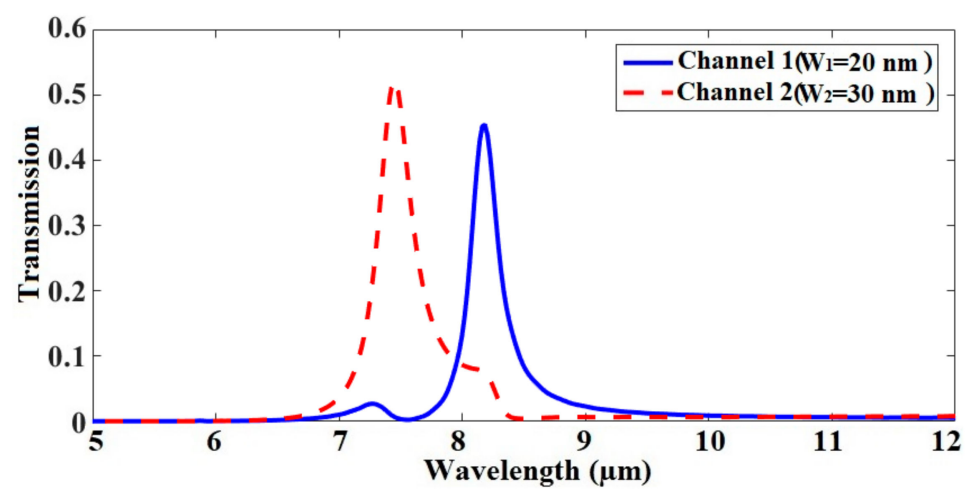

(d)

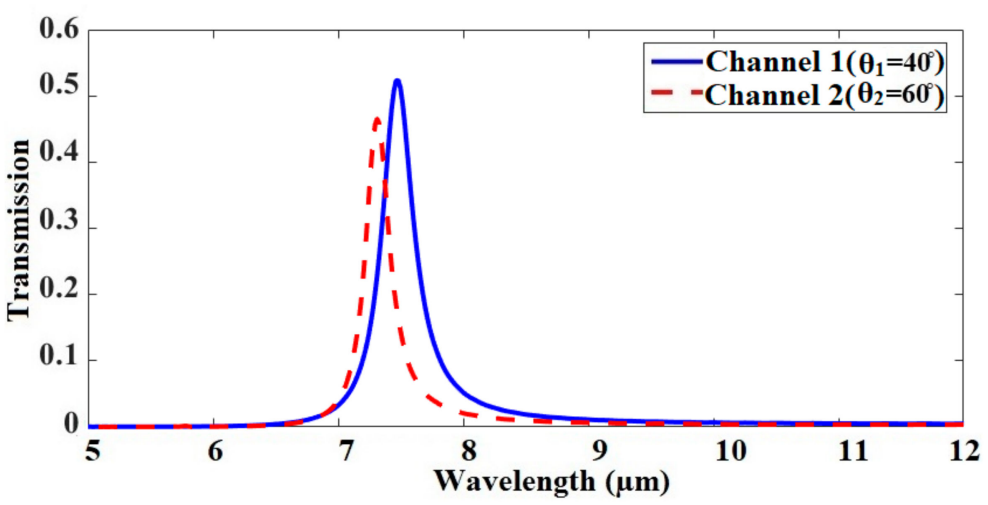

(e)

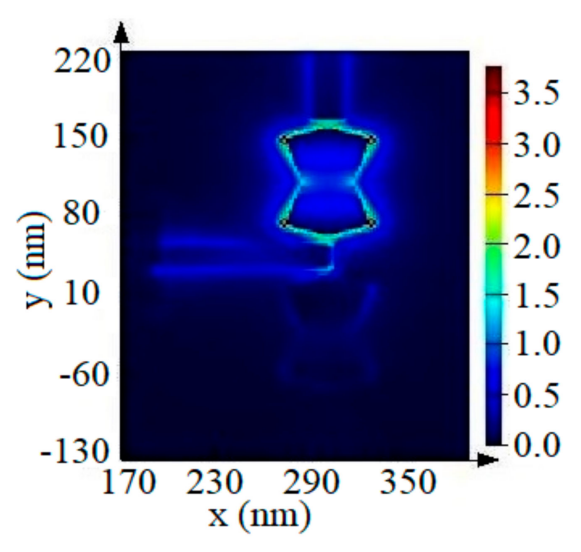

(f)

Figure 4. Cont. 


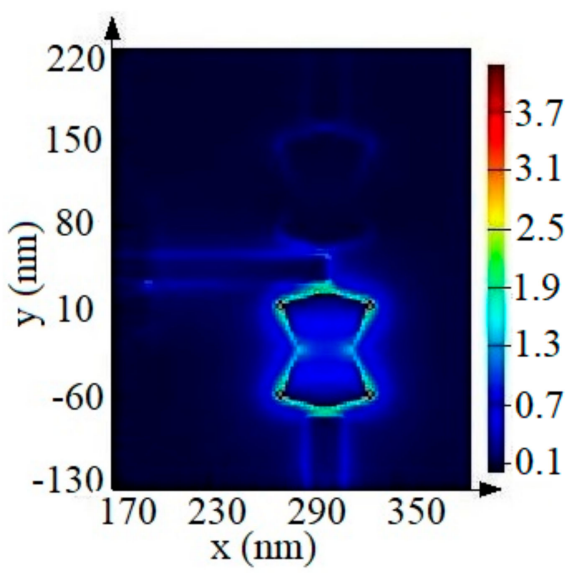

(g)

Figure 4. (a) 2D top view (side view is the same as Figure 1b) of the MIR two-channel demultiplexer. Transmission spectra of the output ports of the proposed two-channel demultiplexer for the resonators with different (b) lengths (channels 1 and 2 with the lengths of $L_{1}=80 \mathrm{~nm}$ and $\mathrm{L}_{2}=100 \mathrm{~nm}$ ), (c) Fermi energies (channels 1 and 2 with the Fermi energies of $E_{\mathrm{f} 1}=0.6 \mathrm{eV}$ and $\mathrm{E}_{\mathrm{f} 2}=0.8 \mathrm{eV}$ ), (d) widths (channels 1 and 2 with the widths of $W_{1}=20 \mathrm{~nm}$ and $W_{2}=30 \mathrm{~nm}$ ), and (e) angles (channel 1 and 2 with the angles of $\theta_{1}=40^{\circ}$ and $\theta_{2}=60^{\circ}$ ). $|E|$ Field profiles of the proposed two-channel demultiplexer for the resonators with different lengths of $L_{1}=80 \mathrm{~nm}$ and $L_{2}=100 \mathrm{~nm}$ in the resonance wavelengths of (f) $\lambda=6.8 \mu \mathrm{m}$ and (g) $\lambda=8.05 \mu \mathrm{m}$.

Cross talk $C$, between the two channels of the proposed demultiplexer of Figure 4a is calculated by [5]

$$
C=10 \log \left(\frac{P_{o}}{P_{i}}\right)
$$

where $P_{i}$ is the power of interested channel and $P_{o}$ is the power of another channel. The crosstalk values of the proposed two-channel demultiplexer of Figure $4 \mathrm{a}$ for different states of Figure $4 \mathrm{~b}-\mathrm{e}$ are given in Table 3.

Table 3. Cross talk values of the proposed demultiplexer in $\mathrm{dB}$

\begin{tabular}{cccc}
\hline Cross Talk (dB) & Channel & $\mathbf{1}$ & $\mathbf{2}$ \\
\hline \multirow{2}{*}{ Figure 4b } & 1 & - & -48.30 \\
\cline { 2 - 4 } & 2 & -33.64 & - \\
\hline \multirow{2}{*}{ Figure 4c } & 1 & - & -25.67 \\
\cline { 2 - 4 } & 2 & -42.24 & - \\
\hline \multirow{2}{*}{ Figure 4d } & 1 & - & -22.38 \\
\cline { 2 - 4 } & 2 & -34.26 & - \\
\hline \multirow{2}{*}{ Figure 4e } & 1 & - & -11.63 \\
\cline { 2 - 4 } & 2 & -7.12 & - \\
\hline
\end{tabular}

The maximum transmission ratio, the full width at half maximum (FWHM), the cross talk, and tunability without need to re-fabricate the structures of some other works are reported and compared with our work in Table 4 . References $[24,40-43,47,48]$ did not report demultiplexing application of their proposed structures, so we leave dash for their cross talks in the table. References [41,42] are absorbers with absorption spectra and they do not contain transmission spectra. Transmission spectra and FWHM are not reported in [44]. FWHMs in [24,26,39,45-47] are not reported and we calculated them approximately. Transmission spectra of [40] contains some ripples and estimation of FWHM is 
not possible. Even though some of the references have better transmission ratio or FWHM than our resonator, the proposed resonator is dedicated for demultiplexing application. Having better cross talk and a wide single mode resonance wavelength range are important parameters for demultiplexing application. These two items are improved in our demultiplexing structure compared to the literature.

Table 4. Comparison of maximum transmission ratios of this work with some others

\begin{tabular}{cccccccccccccccccc}
\hline Ref. & [4] & [5] & [24] & [26] & [39] & [40] & [41] & [42] & [43] & [44] & [45] & [46] & [47] & [48] & This work \\
\hline $\begin{array}{c}\text { Maximum Transmission } \\
\text { Ratio (\%) }\end{array}$ & 80 & 45 & 70 & 50 & 50 & 50 & - & - & 25 & - & 95.8 & 99 & 65 & 92 & 55 \\
\hline Minimum FWHM (nm) & 36 & 59 & 110 & 518 & 300 & - & 300 & 400 & 237 & - & 30 & 2 & 650 & 200 & 220 \\
\hline Crosstalk (dB) & -29 & -30 & - & -47.14 & -38 & - & - & - & - & -14 & -18.9 & -18 & - & - & -48.30 \\
\hline Tunability & no & no & yes & yes & yes & yes & yes & yes & yes & no & no & no & yes & no & yes \\
\hline
\end{tabular}

\section{Conclusions}

In the present study, we have proposed, and analyzed new tunable graphene plasmonic cross-shaped resonator and a two-channel demultiplexer in mid-infrared (MIR) region. It is shown that it is possible to tune the transmission spectrum of the proposed structures by alternating of the applied bias voltage (Fermi energy) of graphene which is an advantage of the proposed structures. Our proposed structures are single mode in the wavelength range of 5-12 $\mu \mathrm{m}$. Based on the proposed plasmonic cross-shaped resonator, two-channel demultiplexer is designed and studied in MIR region. The minimum value of crosstalk for the proposed demultiplexer is $-48.30 \mathrm{~dB}$. The maximum transmission ratio of $55 \%$ and the minimum FWHM of $220 \mathrm{~nm}$ are obtained by our proposed resonator structure. The proposed structures are analyzed by use of three-dimensional finite-difference time-domain (3D-FDTD) method and coupled mode theory (CMT). Our work could be used for further development of nano meter sized active and passive optical devices and structures in MIR region.

Author Contributions: Software and simulation, S.A.; writing-original draft preparation, S.A. and T.F.; writing-review and editing, S.A. and T.F.; All authors discussed the results and contributed to the final manuscript. All authors have read and agreed to the published version of the manuscript.

Funding: This research received no external funding.

Conflicts of Interest: The authors declare no conflict of interest.

\section{References}

1. Lai, W.; Wen, K.; Lin, J.; Guo, Z.; Hu, Q.; Fang, Y. Plasmonic filter and sensor based on a subwavelength end-coupled hexagonal resonator. Appl. Opt. 2018, 57, 6369-6374. [CrossRef] [PubMed]

2. Wang, A.; Dan, Y. Mid-infrared plasmonic multispectral filters. Sci. Rep. 2018, 8, 11257. [CrossRef] [PubMed]

3. Zhao, B.; Huang, Z.; Yang, J.; Zhang, L.; Joshya, R.S.; Guo, C. A high-efficiency multispectral filter based on plasmonic hybridization between two cascaded ultrathin nanogratings. Molecules 2019, 24, 2038. [CrossRef] [PubMed]

4. Danaie, M.; Geravand, A. Design of low-cross-talk metal-insulator-metal plasmonic waveguide intersections based on proposed cross-shaped resonators. J. Nanophotonics 2018, 12, 046009. [CrossRef]

5. Zafar, R.; Chauhan, P.; Salim, M.; Singh, G. Metallic slit-loaded ring resonator-based plasmonic demultiplexer with large crosstalk. Plasmonics 2018, 14, 1013-1017. [CrossRef]

6. Shibayama, J.; Kawai, H.; Yamauchi, J.; Nakano, H. Analysis of a 3D MIM waveguide-based plasmonic demultiplexer using the TRC-FDTD method. Opt. Commun. 2019, 452, 360-365. [CrossRef]

7. Sharma, A.K.; Pandey, A.K. Self-referenced plasmonic sensor with $\mathrm{TiO}_{2}$ grating on thin Au layer: Simulated performance analysis in optical communication band. J. Opt. Soc. Am. B 2019, 36, F25-F31. [CrossRef]

8. Cheng, Y.C.; Chang, Y.J.; Chuang, Y.C.; Huang, B.Z.; Chen, C.C. A plasmonic refractive index sensor with an ultrabroad dynamic sensing range. Sci. Rep. 2019, 9, 5134. [CrossRef] 
9. Islam, M.S.; Sultana, J.; Rifat, A.A.; Ahmed, R.; Dinovitser, A.; Ng, B.W.-H.; Heidepriem, H.E.; Abbott, D. Dual-polarized highly sensitive plasmonic sensor in the visible to near-IR spectrum. Opt. Express 2018, 26, 30347-30361. [CrossRef]

10. Jaber, M.S.; Tawfeeq, S.K.; Fyath, R.S. Design investigation of $4 \times 4$ nonblocking hybrid plasmonic electrooptic switch. Photonics 2019, 6, 47. [CrossRef]

11. Nurmohammadi, T.; Abbasian, K.; Yadipour, R. Ultra-fast all-optical plasmon induced transparency in a metal-insulator-metal waveguide containing two Kerr nonlinear ring resonators. J. Opt. 2018, 20, 055504. [CrossRef]

12. Chauhan, D.; Mola, G.T.; Dwivedi, R.P. An ultra-compact plasmonic modulator/switch using $\mathrm{VO}_{2}$ and elasto-optic effect. Optik 2019, 201, 163531. [CrossRef]

13. Li, S.P.; Liu, H.J.; Sun, Q.B.; Huang, N. Multi-channel terahertz wavelength division demultiplexer with defects-coupled photonic crystal waveguide. J. Mod. Opt. 2016, 63, 955-960. [CrossRef]

14. Dai, D.X.; Wang, J.; Chen, S.T.; Wang, S.P.; He, S.L. Monolithically integrated 64-channel silicon hybrid demultiplexer enabling simultaneous wavelength- and mode-division-multiplexing. Laser Photonics Rev. 2015, 18, 339-344. [CrossRef]

15. Zhang, L.; Tang, L.L.; Wei, W.; Cheng, X.L.; Wang, W.; Zhang, H. Enhanced near-infrared absorption in graphene with multilayer metal-dielectric-metal nanostructure. Opt. Express 2016, 24, 20002-20009. [CrossRef] [PubMed]

16. Azzazi, A.; Swillam, M.A. Nanoscale highly selective plasmonic quad wavelength demultiplexer based on a metal-insulator-metal. Opt. Commun. 2015, 344, 106-112. [CrossRef]

17. Rodrigo, D.; Limaj, O.; Janner, D.; Etezadi, D.; de Abajo, F.J.G.; Pruneri, V.; Altug, H. Mid-infrared plasmonic biosensing with graphene. Science 2015, 349, 165-168. [CrossRef]

18. Woessner, A.; Lundeberg, M.B.; Gao, Y.; Principi, A.; Alonso-Gonzaolez, P.; Carrega, M.; Watanabe, K.; Taniguchi, T.; Vignale, G.; Polini, M.; et al. Highly confined low-loss plasmons in graphene-boron nitride heterostructures. Nat. Mater. 2015, 14, 421-425. [CrossRef]

19. Bonaccorso, F.; Sun, Z.; Hasan, T.; Ferrari, A.C. Graphene photonics and optoelectronics. Nat. Photonics 2010, 4, 611-622. [CrossRef]

20. Wang, F.; Qin, C.Z.; Wang, B.; Long, H.; Wang, K.; Lu, P.X. Rabi oscillations of plasmonic super modes in graphene multilayer arrays. IEEE J. Sel. Top. Quantum Electron. 2017, 23, 4600105. [CrossRef]

21. Grigorenko, A.N.; Polini, M.; Novoselov, K.S. Graphene plasmonics. Nat. Photonics 2012, 6, 749-758. [CrossRef]

22. Low, T.; Avouris, P. Graphene plasmonics for terahertz to midinfrared applications. ACS Nano 2014, 8, 1086-1101. [CrossRef] [PubMed]

23. Zhou, L.; Tan, Y.L.; Wang, J.Y.; Xu, W.C.; Yuan, Y.; Cai, W.S.; Zhu, S.N.; Zhu, J. 3D self-assembly of aluminium nanoparticles for plasmon enhanced solar desalination. Nat. Photonics 2016, 10, 393-398. [CrossRef]

24. Asgari, S.; Rajabloo, H.; Granpayeh, N.; Oraizi, H. Tunable graphene-based mid-infrared band-pass planar filter and its application. Chin. Phys. B 2018, 27, 084212. [CrossRef]

25. Moazami, A.; Hashemi, M.; Shirazi, N.C. High efficiency tunable graphene-based plasmonic filter in the THz frequency range. Plasmonics 2019, 14, 359-363. [CrossRef]

26. Asgari, S.; Granpayeh, N. Applications of tunable nanoscale midinfrared graphene based slot cavity in nanophotonic integrated circuits. IEEE Trans. Nanotechnol. 2018, 17, 533-542. [CrossRef]

27. Asgari, S.; Granpayeh, N.; Kashani, Z.G. Plasmonic mid-infrared wavelength selector and linear logic gates based on graphene cylindrical resonator. IEEE Trans. Nanotechnol. 2018, 18, 42-50. [CrossRef]

28. Zhou, F.; Du, W. Ultrafast all-optical plasmonic graphene modulator. Appl. Opt. 2018, 57, 6645-6650. [CrossRef]

29. Zhu, Y.; Deng, C.; Huang, L.; Hu, G.; Yun, B.; Zhang, R.; Cui, Y. Hybrid plasmonic graphene modulator with buried silicon waveguide. Opt. Commun. 2020, 456, 124559. [CrossRef]

30. Wu, T.; Luo, Y.; Maier, S.A.; Wei, L. Phase-matching and peak nonlinearity enhanced third-harmonic generation in graphene plasmonic coupler. Phys. Rev. Appl. 2019, 11, 014049. [CrossRef]

31. Bahadori-Haghighi, S.; Ghayour, R.; Sheikhi, M.H. Design and analysis of low loss plasmonic waveguide and directional coupler based on pattern-free suspended graphene sheets. Carbon 2018, 129, 653-660. [CrossRef] 
32. Wu, P.; Chen, Z.; Xu, D.; Zhang, C.; Jian, R. A Narrow Dual-Band Monolayer Unpatterned Graphene Based Perfect Absorber with Critical Coupling in the Near Infrared. Micromachines 2020, 11, 58. [CrossRef]

33. Cen, C.; Chen, Z.; Xu, D.; Jiang, L.; Chen, X.; Yi, Z.; Wu, P.; Li, G.; Yi, Y. High Quality Factor, High Sensitivity Metamaterial Graphene-Perfect Absorber Based on Critical Coupling Theory and Impedance Matching. Nanomaterials 2020, 10, 95. [CrossRef] [PubMed]

34. Houshmand, M.; Hossein Zandi, M.; Gorji, N.E. Modeling of optical losses in graphene contacted thin film solar cells. Mat. Lett. 2016, 164, 493-497. [CrossRef]

35. Yu, X.; Li, Y.; Hu, X.; Zhang, D.; Tao, Y.; Liu, Z.; He, Y.; Haque, M.A.; Liu, Z.; Wu, T.; et al. Narrow bandgap oxide nanoparticles coupled with graphene for high performance mid-infrared photodetection. Nat. Commun. 2018, 9, 4299. [CrossRef] [PubMed]

36. Deng, T.; Zhang, Z.; Liu, Y.; Wang, Y.; Su, F.; Li, S.; Zhang, Y.; Li, H.; Chen, H.; Zhao, Z.; et al. Three-Dimensional Graphene Field-Effect Transistors as High-Performance Photodetectors. Nano Lett. 2019, 19, 1494-1503. [CrossRef]

37. Yarahmadi, M.; Moravvej-Farshi, M.K.; Yousefi, L. Subwavelength Graphene-Based Plasmonic THz Switches and Logic Gates. IEEE Trans. Terahertz Sci. Technol. 2015, 5, 725-731. [CrossRef]

38. Wang, X.; Chen, C.; Pan, L.; Wang, J. A graphene-based Fabry-Pérot spectrometer in mid-infrared region. Sci. Rep. 2016, 6, 32616. [CrossRef]

39. Asgari, S.; Granpayeh, N. Tunable plasmonic dual wavelength multi/demultiplexer based on graphene sheets and cylindrical resonator. Opt. Commun. 2017, 393, 5-10. [CrossRef]

40. Feng, Y.; Liu, Y.; Shi, Y.; Teng, J. Tunable plasmonic filter based on graphene-layered waveguide. Mod. Phys. Lett. B 2018, 32, 1850110. [CrossRef]

41. Li, H.J.; Wang, L.L.; Zhai, X. Tunable graphene-based mid-infrared plasmonic wide-angle narrowband perfect absorber. Sci. Rep. 2016, 6, 36651. [CrossRef] [PubMed]

42. Goldflam, M.D.; Fei, Z.; Ruiz, I.; Howell, S.W.; Davids, P.S.; Peters, D.W.; Beechem, T.E. Designing graphene absorption in a multispectral plasmon-enhanced infrared detector. Opt. Express 2017, 25, 12400-12408. [CrossRef] [PubMed]

43. Li, H.J.; Wang, L.L.; Sun, B.; Huang, Z.R.; Zhai, X. Controlling midinfrared surface plasmon polaritons in the parallel graphene pair. Appl. Phys. Express 2014, 7, 125101. [CrossRef]

44. Nakayama, K.; Tonooka, Y.; Ota, M.; Ishii, Y.; Fukuda, M. Passive plasmonic demultiplexers using multimode interference. IEEE J. Lightwave Technol. 2018, 36, 1979-1984. [CrossRef]

45. Azar, M.T.H.; Zavvari, M.; Arashmehr, A.; Zehforoosh, Y.; Mohammadi, P. Design of a high-performance metal-insulator-metal plasmonic demultiplexer. J. Nanophotonics 2017, 11, 026002. [CrossRef]

46. Talebzadeh, R.; Soroosh, M.; Kavian, Y.S.; Mehdizadeh, F. Eight-channel all-optical demultiplexer based on photonic crystal resonant cavities. Optik 2017, 140, 331-337. [CrossRef]

47. Feng, Y.; Liu, Y.; Wang, X.; Dong, D.; Shi, Y.; Tang, L. Tunable Multichannel Plasmonic Filter Based on a Single Graphene Sheet on a Fibonacci Quasiperiodic Structure. Plasmonics 2018, 13, 653-659. [CrossRef]

48. IM, C.S.; Lee, S.S. Highly efficient and angle-tolerant mid-infrared filter based on a cascaded etalon resonator. Opt. Express 2017, 25, 16083-16091. [CrossRef]

49. Janfaza, M.; Mansouri-Birjandi, M.A.; Tavousi, A. Tunable plasmon-induced reflection based on graphene nanoribbon Fabry- Perot resonator and nanodisks. Opt. Mater. 2018, 84, 675-680. [CrossRef]

50. Janfaza, M.; Mansouri-Birjandi, M.A.; Tavousi, A. Dynamic switching between single and double plasmon induced reflection through graphene nanoribbons based structure. Mater. Res. Express 2018, 5, 115022. [CrossRef]

51. Sreekanth, K.V.; Alapan, Y.; ElKabbash, M.; Ilker, E.; Hinczewski, M.; Gurkan, U.A.; De Luca, A.; Strangi, G. Extreme sensitivity biosensing platform based on hyperbolic metamaterials. Nat. Mater. 2016, 15, 621-627. [CrossRef]

52. Janfaza, M.; Mansouri-Birjandi, M.A.; Tavousi, A. Tunable plasmonic band-pass filter based on Fabry-Perot graphene nanoribbons. Appl. Phys. B 2017, 123, 262. [CrossRef]

53. Vakil, A.; Engheta, N. Transformation optics using graphene. Science 2011, 32, 1291-1294. [CrossRef] [PubMed]

54. Li, Q.; Wang, T.; Su, Y.; Yan, M.; Qiu, M. Coupled mode theory analysis of mode-splitting in coupled cavity system. Opt. Express 2010, 18, 8367-8382. [CrossRef] [PubMed] 
55. Feng, Y.; Liu, Y.; Shi, Y.; Wang, X.; Dong, D. An ultra-compact tunable intersection structure based on graphene nanoribbon. J. Phys. D Appl. Phys. 2017, 50, 185101. [CrossRef]

56. Ke, S.; Wang, B.; Huang, H.; Long, H.; Wang, K.; Lu, P. Plasmonic absorption enhancement in periodic cross-shaped graphene arrays. Opt. Express 2015, 23, 8888-8900. [CrossRef] [PubMed]

57. Li, H.J.; Wang, L.L.; Sun, B.; Huang, Z.R.; Zhai, X. Tunable mid-infrared plasmonic band-pass filter based on a single graphene sheet with cavities. J. Appl. Phys. 2014, 116, 224505. [CrossRef]

58. $\mathrm{Su}, \mathrm{W}$. A four-port ultra-compact terahertz splitting filter based on graphene nanoribbon. IEEE PhotonicS Technol. Lett. 2019, 31, 86-89. [CrossRef]

59. Nikitin, A.; Guinea, F.; Garcła-Vidal, F.; Martłn-Moreno, L. Edge and waveguide terahertz surface plasmon modes in graphene microribbons. Phys. Rev. B 2011, 84, 161407. [CrossRef]

60. Tian, J.; Li, J. Investigation on plasmon induced transparency and its application in an MIM type compound plasmonic waveguide. Prog. Electromagn. Res. C 2020, 98, 199-212. [CrossRef]

(C) 2020 by the authors. Licensee MDPI, Basel, Switzerland. This article is an open access article distributed under the terms and conditions of the Creative Commons Attribution (CC BY) license (http://creativecommons.org/licenses/by/4.0/). 